\title{
A EQUIPE DE ENFERMAGEM DENTRO DA ÁREA HOSPITALAR E OS ATMB: PERFIL SOCIODEMOGRÁFICO E CATEGORIA
}

\section{Grasiela Aparecida Coura Querobino Alvarenga ${ }^{1}$; Heitor Cardoso de Brito ${ }^{2}$; Marileny Boechat Frauches ${ }^{3}$}

${ }^{1}$ Mestranda do Programa de Mestrado em Gestão Integrada do Território, Universidade Vale do Rio Doce. Professora do Instituto Federal de Minas Gerais (IFMG), (grasiela.alvarenga@ifmg.edu.br), Governador Valadares, Minas Gerais, Brasil.

${ }^{2}$ Mestrando do Curso da Pós-graduação em Tecnologia, Ambiente e Sociedade da Universidade Federal dos Vales do Jequitinhonha e Mucuri (UFVJM), Teófilo Otoni, MG. Professor do Instituto Federal de Minas Gerais (IFMG), Governador ValadaresMG.

${ }^{3}$ Professora doutora do Programa de Mestrado em Gestão Integrada do Território da Universidade Vale do rio Doce, Governador Valadares-MG.

Recebido em: 22/09/2018 - Aprovado em: 23/11/2018 - Publicado em: 03/12/2018 DOI: $10.18677 /$ EnciBio_2018B90

O ambiente hospitalar é o principal local de ocorrência de acidentes de trabalho com exposição a material biológico (ATMB), assim destaca-se que a equipe da enfermagem é considerada dentro da área da saúde, a ocupação mais acometida por tais acidentes. Isso se deve a prática diversificada de procedimentos, jornada de trabalho extensa, exposição a diferentes riscos biológicos e por exercer atividades em contato direto com pacientes. Assim, pode-se considerar que o ATMB é um problema de Saúde Pública. O objetivo foi identificar o perfil sociodemográfico e a categoria profissional dentro da equipe da enfermagem que mais é acometida por ATMB no ambiente hospitalar. Tratou-se de um levantamento bibliográfico retrospectivo, dos últimos 10 anos, de artigos publicados na íntegra, em português, indexados nas bases de dados LILACS e SciELO, utilizando como descritores: acidente de trabalho com exposição a material biológico, equipe da enfermagem, perfil sociodemográfico. Para esta pesquisa foram selecionados a partir dos critérios de inclusão/exclusão 28 artigos. Observou-se que a maioria dos profissionais da equipe da enfermagem era do sexo feminino, na faixa etária entre 20 a 50 anos; assim tornam-se mais vulneráveis a ocorrência de agravos como, doenças ocupacionais e ATMB. Os técnicos e auxiliares de enfermagem foram as categorias mais acometidas por ATMB em função da maior incidência na equipe da enfermagem dentro do ambiente hospitalar. Assim medidas preventivas devem ser implantadas como a educação continuada, práticas seguras, palestras sobre biossegurança e cursos motivacionais serão medidas educativas para minimizar e/ou eliminar a ocorrência dos ATMB.

PALAVRAS-CHAVE: acidente de trabalho com exposição a material biológico, equipe da enfermagem, perfil sociodemográfico. 


\title{
THE NURSING TEAM WITHIN THE HOSPITAL AREA AND THE ATMB: SOCIODEMOGRAPHIC PROFILE AND CATEGORY
}

\begin{abstract}
The hospital environment is the main place of occurrence of work accidents with exposure to biological material (ATMB), so it is highlighted that the nursing team is considered within the health area, the occupancy most affected by such accidents. This is due to the diversified practice of procedures, extensive working hours, exposure to different biological risks, and to perform activities in direct contact with patients. Thus, it can be considered that ATMB is a Public Health problem. The objective was to identify the sociodemographic profile and the professional category within the nursing team that is most affected by ATMB in the hospital environment. This was a retrospective bibliographical survey of the last 10 years of articles published in full, in Portuguese, indexed in the LILACS and SciELO databases, using as descriptors: work accident with exposure to biological material, nursing team, profile sociodemographic. For this research, 28 articles were selected from the inclusion / exclusion criteria. It was observed that the majority of the professionals of the nursing team were female, in the age group between 20 and 50 years; they become more vulnerable to the occurrence of diseases such as occupational diseases and ATMB. Nursing technicians and auxiliaries were the categories most affected by ATMB due to the higher incidence in the nursing team within the hospital environment. Thus preventive measures should be implemented as continuing education, safe practices, lectures on biosafety and motivational courses will be educational measures to minimize and / or eliminate the occurrence of ATMB.
\end{abstract}

KEYWORDS: work accident with exposure to biological material, nursing team, sociodemographic profile.

\section{INTRODUÇÃO}

Nos últimos anos a Organização Internacional do Trabalho (OIT) registrou que mais de 2,3 milhões de trabalhadores morrem por acidentes de trabalho e que 860 mil sofrem ferimentos diariamente no mundo. Os custos globais, diretos ou indiretos, chegam a sete trilhões de reais. Ressalta-se que o número de mortes ocupacionais no mundo são maiores do que as ocorridas em guerras (OIT, 2018).

No Brasil este índice é significativamente preocupante para a estatística mundial, pois mais de 700 mil acidentes de trabalho são registrados por ano, sendo o país o quarto colocado no ranking mundial. De acordo com o Anuário Estatístico de Acidente de Trabalho (AEAT) nos anos de 2013, 2014 e 2015 foram mais de dois milhões de acidentes de trabalho em todo o Brasil, sendo o maior índice encontrado na região Sudeste (mais de um milhão) e o menor índice na região Norte (mais de 90 mil) (BRASIL, 2016).

De acordo com estes índices apresentados sobre os acidentes de trabalho no mundo e no Brasil, destaca-se que os acidentes que envolvem exposição a material biológico (ATMB) apresenta alta prevalência no Brasil; sendo mais de 130 mil acidentes por ano. Estes tiveram a maior ocorrência nas áreas da saúde, consequentemente dentro dos hospitais; ocupando o segundo lugar nas estatísticas e, perdendo somente para as indústrias e empresas de obras civis (BRASIL, 2015).

O ATMB é considerado um problema de Saúde Pública, sendo preocupante para os empregadores e para o sistema previdenciário de todo país devido aos seus efeitos nas vítimas deste tipo de acidente. Além disso, o ATMB pode gerar prejuízos econômicos e sociais, o trabalhador acidentado precisa afastar-se das suas 
atividades produtivas (REIS et al, 2013; GIANCOTTI et al, 2014; BRASIL, 2014; ARANTES et al., 2017).

Segundo Nogueira et al., (2016) tanto em países desenvolvidos, quanto em países em desenvolvimento, os acidentes representam um dos maiores agravos à saúde dos trabalhadores. Considerado como uma das maiores preocupações para as instituições, desta forma constitui um tema de relevância para pesquisas relacionadas à saúde do trabalhador no Brasil.

Em função de diversas questões que envolvem a saúde do trabalhador, vêm ocorrendo na comunidade científica um grande interesse e preocupação com estes acidentes que envolvem principalmente os profissionais da saúde. Dentre os diversos riscos laborais a que estão expostos, o que envolve acidente de trabalho com exposição a material biológico (ATMB) é um dos mais relevantes (BARBOSA et al., 2009; OLIVEIRA; GONÇALVES, 2010; REIS et al., 2013; CAVALCANTE et al., 2013; GIANCOTTI, et al., 2014).

Desta forma, considera-se que ATMB aqueles que envolvem sangue e outros fluidos orgânicos ocorridos nos profissionais da saúde durante o exercício de suas atividades, os quais estão diariamente expostos a materiais biológicos potencialmente contaminados. Os ATMB são capazes de transmitir mais de 20 tipos de patógenos diferentes, como hepatite $B(\mathrm{HBV})$, hepatite $\mathrm{C}(\mathrm{HCV})$ e o vírus da imunodeficiência humana (HIV) (SINAN, 2011).

O ambiente hospitalar é o principal estabelecimento de ocorrência de ATMB, por se tratar de um lugar onde acontecem atividades complexas levando a contato com procedimentos invasivos (ARANTES et al., 2017). Assim destaca-se a importância de adoção de medidas preventivas para a proteção dos profissionais da saúde que atuam nestes locais, por meio de ações preventivas, educativas e fiscalizadoras, com o objetivo de minimizar ou eliminar tais ocorrências (ARAÚJO et al., 2012).

Os profissionais de saúde que exercem atividades nas áreas de operação, prestação de cuidados, salas cirúrgicas, emergência, e laboratórios têm um risco maior de exposição, como também os profissionais da limpeza, de resíduos e coletores, sendo que as atividades são de manipulação de materiais contaminados (MACHADO; ASSUNÇÃO, 2012).

Os estabelecimentos hospitalares ofertam serviços à saúde, no sentido de atender, curar e tratar pacientes com diversas patologias. Mas é também um ambiente que sujeita os profissionais da equipe de enfermagem a riscos ambientais que podem acarretar ATMB e doenças ocupacionais. Entre os diversos profissionais da saúde que operam na assistência hospitalar a equipe da enfermagem tem a maior representatividade (BARBOZA et al., 2004).

Assim sendo, os profissionais de enfermagem é uma categoria que se expõe a uma série de riscos, consequentemente é a que tem a maior probabilidade de ocorrência de agravos como, doenças ocupacionais e ATMB (RAFFONE; HENNINGTON, 2005; BARBOSA et al., 2009).

O hospital é um dos locais onde os profissionais da equipe de enfermagem permanecem em maior parte da sua vida cotidiana, e na maioria das vezes cumprem mais que um turno de trabalho; buscando salvar vidas e restaurando a saúde de muitos pacientes. Estes ambientes têm exposto os profissionais a doenças em função das atividades laborais, e as instituições aparentemente não estão preocupadas com a proteção e muito menos com a promoção e manutenção da saúde destes trabalhadores (PEDROZA, 2014). 
A equipe da enfermagem é considerada dentro da área da saúde, a ocupação que mais é acometida por ATMB. Isto se deve a fatores como, prática de procedimentos diversos e complexos, jornada de trabalho extensa, exposição a diferentes riscos que podem interferir diretamente na qualidade da assistência e do trabalho destes profissionais (BARBOSA et al., 2009; ARANTES, 2017).

Diversos estudos na literatura evidenciaram que a equipe da enfermagem se torna muito suscetível a ocorrência de ATMB envolvendo material perfurocortante (BAKKE; ARAÚJO, 2010; OLIVEIRA;GONÇALVES, 2010; DONATELLI et al., 2015). Isto ocorre devido a realização de procedimentos invasivos, tais como, a manipulação diária de agulhas e escalps, administração de medicamentos subcutâneos, procedimentos de punção venosa e soroterapia. A maioria destes acidentes ocorrem em contato com a pele, mucosa com sangue e secreções; e a maioria dos ATMB ocorre durante a prática de procedimentos a beira do leito, ou nos postos de enfermagem durante o preparo de medicamentos, depois no descarte de excretas ou durante $o$ atendimento de pacientes obesos e inquietos (BARBOSA et al., 2009; BAKKE; ARAÚJO, 2010).

As principais causas dos ATMB podem estar relacionadas à agitação e agressividade dos pacientes e manuseio inadequado de materiais ou máquinas/equipamentos (NOWAK et al., 2013). Segundo Alves (2011), outros fatores ainda podem ser destacados, como descuido do profissional na realização de atividades, carga de trabalho extensiva, baixa remuneração, jornada longa de trabalho (seja em casa ou na empresa), estrutura física da empresa e o processo de trabalho.

Os ATMB trazem sérias consequências para a vida do profissional, sua família, chefes e pessoas que estão em sua convivência social, comprometendo assim a qualidade e expectativa de vida deste profissional. Em relação a problemas para a empresa, estes geram prejuízos na qualidade da assistência prestada aos pacientes e também o afastamento do profissional qualificado, que deverá treinar outro para trabalhar em seu lugar (MARZIALE et al., 2014).

Outras consequências quanto aos ATMB, segundo Saliba (2015) são as obrigatoriedades que o empregador deverá arcar quando ocorrido, sendo: estabilidade provisória de um ano quando o profissional da equipe da enfermagem tiver a necessidade de afastamento de mais de 15 dias pelo Instituto Nacional do Seguro Social (INSS); responsabilidade civil garantindo a percepção de indenização se ocorrer culpa do empregador e; responsabilidade penal ou criminosa em caso de homicídio culposo pelos gerentes, supervisores, encarregados e os profissionais do Serviço Especializado em Engenharia de Segurança e em Medicina do Trabalho (SESMT).

Portanto para eliminar estas possíveis consequências, medidas preventivas devem ser implantadas com a finalidade de prevenir e controlar os riscos biológicos relativos ao ambiente de trabalho e ao profissional. As medidas podem ser relativas ao ambiente de trabalho, que podem ser aplicadas na fonte ou na trajetória, estas devem ser prioritárias; medidas administrativas, como limitação de tempo de exposição ao risco do profissional, educação, treinamentos e exames médicos; e por último o uso de equipamento de proteção individual (EPI) (quando não é possível o controle coletivo ou administrativo) (SALIBA, 2013). Para Marziale et al. (2014), a utilização de procedimentos alternativos e de tecnologia em dispositivos e materiais médico-hospitalares, como os dispositivos de segurança (agulha com dispositivo de segurança) exigidos no Brasil pela NR-32, podem minimizar o acometimento de ATMB. 
Vale ressaltar que uma das formas ou medidas para diminuir e/ou eliminar os ATMB é o investimento em prevenção, pode gera custos, mas é uma das principais atitudes que as empresas e o empregador devem contribuir. Assim, pode-se realizar medidas por meio de fiscalizações preventivas, treinamentos de acordo com o tipo de atividade realizada e campanhas de conscientização para todos os profissionais, as quais auxiliarão na inserção da cultura de prevenção às futuras gerações e que será capaz de reverter esse quadro preocupante no Brasil (SALIBA, 2017).

Outras sugestões de prevenção e medidas para amenizar os ATMB é a implantação da Norma Regulamentadora 32 (NR-32) dentro das instituições hospitalares, que trata da Segurança e Saúde no trabalho em serviços de saúde e dos Riscos Biológicos, publicada em 2005 e que estabelece nos locais de saúde as diretrizes básicas de medidas de proteção a segurança e a saúde de trabalhadores (BRASIL, 2005). A imunização também é considerada uma proteção contra algumas doenças; como a hepatite $\mathrm{B}$, que é uma doença imunoprevenível, e o esquema vacinal devem ser completados antecipadamente por três doses. Mas observa-se que muitos profissionais da saúde não estão protegidos suficientemente, ou por não completarem o esquema vacinal ou por não apresentarem resposta vacinal (CAVALCANTE et al., 2013).

Desta forma foi realizado um levantamento bibliográfico retrospectivo, dos últimos 10 anos de artigos publicados na íntegra, idioma em português, indexados nas bases de dados LILACS (Centro Latino-americano e do Caribe de informação em Ciências da Saúde), SciELO (Scientific Eletronic Library Online), utilizando como descritores: acidente de trabalho com exposição a material biológico, equipe da enfermagem, perfil sociodemográfico. A busca e análise de dados provenientes de artigos científicos foram realizadas em agosto de 2018.

No levantamento bibliográfico foram encontrados 28 artigos que atendiam aos critérios de inclusão/exclusão. A partir da análise dos artigos foi possível identificar o perfil sociodemográfico e a categoria profissional da equipe da enfermagem que mais foi acometida por acidente de trabalho com exposição a material biológico (ATMB) dentro de hospitais.

Diante deste contexto, buscou-se por meio de uma revisão da literatura, identificar o perfil sociodemográfico e a categoria profissional dentro da equipe da enfermagem que mais é acometida por acidente de trabalho com exposição a material biológico no ambiente hospitalar.

\section{Perfil socioeconômico e demográfico da equipe da enfermagem}

A maioria dos ATMB ocorre com o gênero feminino, sendo uma tendência a feminização das classes da área da saúde, principalmente dentro da equipe da enfermagem, observada em vários estudos (KON et al., 2011; ARAÚJO et al., 2012; MARTINS et al., 2014; DONATELLI et al., 2015; NEGRINHO et al., 2017). Martins et al., (2014) explicam que esta predominância do sexo feminino na área da saúde se deve ao aumento do grau de escolaridade e ao ingresso nas universidades; assim consequentemente maiores ocorrências com ATMB por serem a maioria nas redes de saúde.

Tal composição corrobora com a ideia de feminilização da enfermagem resultante da história e cultura da profissão. O cuidado com a saúde foi muito associado ao cuidado materno, doméstico, doentes e idosos, oferecido pela mulher com práticas que se estabeleceram e foram transmitidas ao longo das gerações. Também é atribuído a questões relacionadas à formação profissional e a ideia empírica de que o homem pode não ter habilidades e competências para algumas 
formas de cuidado, o processo de recrutamento que seleciona mais mulheres que homens e ainda questões relacionadas à organização do espaço de trabalho e política administrativa das instituições de saúde (MACHADO et al., 2015).

A maior parte dos registros com ATMB ocorrem com o sexo feminino, refletindo a trajetória que a equipe da enfermagem tem passado nos últimos anos; que predominantemente a maioria são formadas por mulheres (BARROS et al., 2016).

Este estudo verificou a predominância do sexo feminino envolvido nos ATMB em todos os artigos analisados, muitos justificaram pela composição da equipe de enfermagem ser majoritariamente por este gênero; outros relataram que historicamente a enfermagem é caracterizada como uma profissão quase que exclusiva feminina, onde a mulher tem o desejo e o dom de cuidar, prevenir e promover a saúde dos pacientes (BARBOZA et al., 2004; SILVA et al., 2010).

Com relação à faixa etária destes profissionais, registra-se uma população trabalhadora jovem, e que têm menos de 40 anos (BAKKE; ARAÚJO, 2010; MARTINS et al., 2014; DONATELLI et al., 2015; BARROS et al., 2016; NEGRINHO et al., 2017; PAIVA et al., 2017).

De acordo com os estudos sobre ATMB com profissionais da equipe da enfermagem a faixa etária mais acometida esta entre 20 a 40 anos de idade conduzidos por Silva et al. (2009), Bakke e Araújo (2010), Rios et al. (2012), Donatelli et al., 2015 e Arantes et al. (2017). Enquanto que Reis et al. (2013) mostraram uma maior ocorrência na faixa etária entre 22 a 25 anos, e Giancotti et al. (2014) observaram uma faixa maior entre 20 a 50 anos. Pode-se observar diante destes dados que os ATMB tem ocorrido significamente entre os profissionais em idade produtiva, ou seja, no período potencial da mão-de-obra, assim torna-se o número maior de profissionais nesta idade nas instituições de saúde (ARANTES et al. 2017).

Outro aspecto observado foi em relação ao número de horas semanais trabalhadas pelos profissionais de enfermagem. Conforme figura 1, 40,0\% destes profissionais trabalham até 40 horas semanais, 31,9\% trabalham de 41 a 60 horas e $14,3 \%$ chega a fazer mais de 60 horas semanais. A jornada de trabalho tem-se demonstrado como fator estressor no trabalho, já tendo sido relacionada à depressão e Síndrome de Burnout (MACHADO et al., 2015). Bezerra de Lima et al. (2013) e Machado et al. (2015) identificaram o ritmo de trabalho, além das responsabilidades próprias da função que são fatores estressores, como também a sobre carga de trabalho, movimentos repetitivos; estes são fortes causadores das ocorrências de ATMB, pois os profissionais da enfermagem algumas vezes, apresentam dupla ou mais jornadas de trabalho. A figura 1 abaixo mostra a proporção em porcentagem de profissionais da equipe da enfermagem no Brasil que realizam vários tipos de jornada de trabalho, realizando em vários tipos de instituição de saúde. 


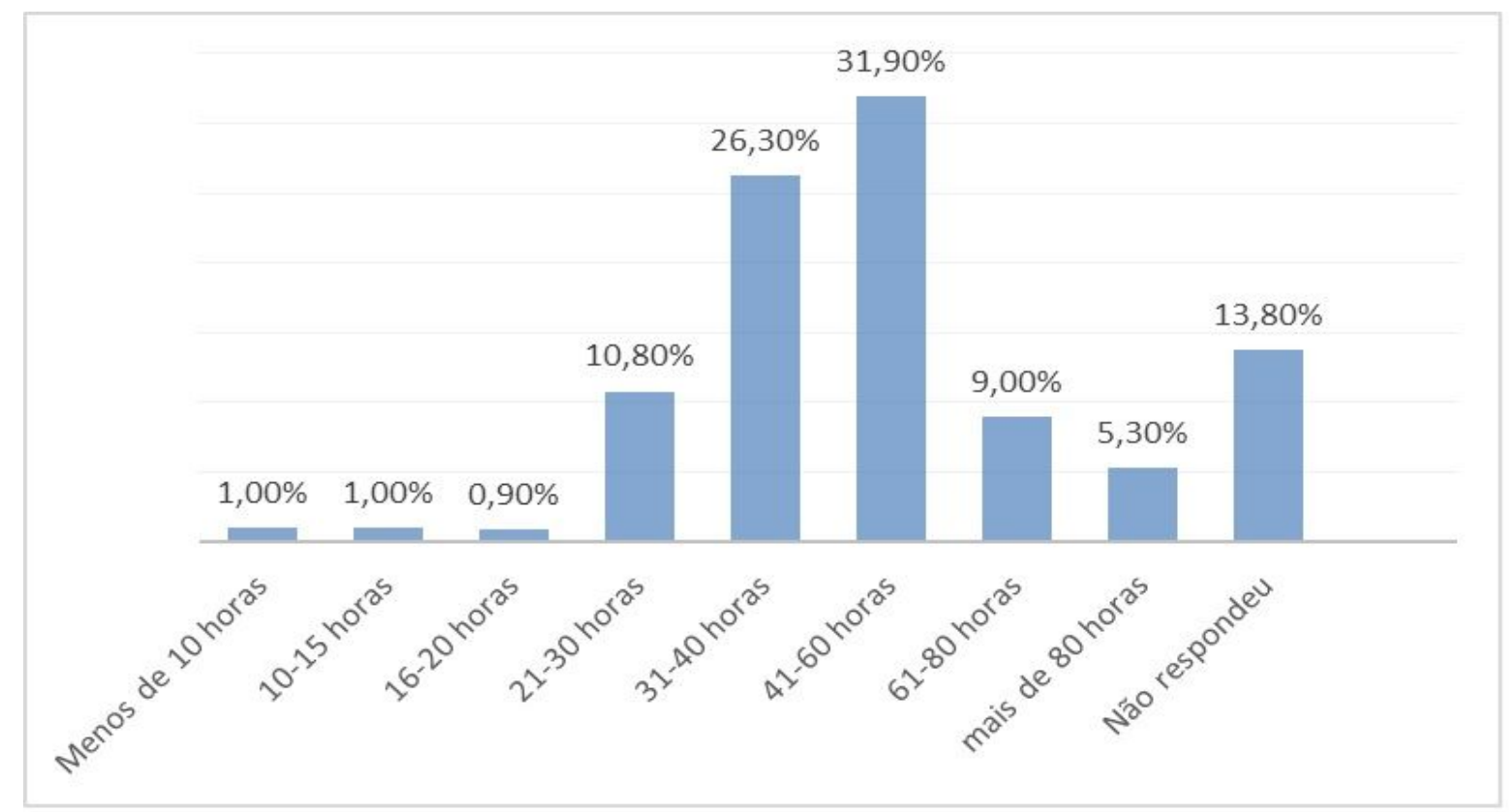

FIGURA 1: Número de horas semanais trabalhadas pelos profissionais da equipe da enfermagem. Fonte: Pesquisa Perfil da Enfermagem no Brasil - (MACHADO et al., 2015).

A jornada de trabalho acentuada tem sido correlacionada à escassez de profissionais capacitados. Felli (2012) estimou que há uma média de sete enfermeiros a cada 100 leitos nos hospitais do Brasil, e que este número de trabalhadores não é suficiente para atendimento da demanda; mantendo um célere ritmo de trabalho, acentuado desgaste profissional, interferindo diretamente na saúde ocupacional e qualidade da assistência. Diante dos artigos analisados, os ATMB têm ocorrido com os profissionais da equipe da enfermagem que laboram suas atividades acima de 40 horas semanais, demonstrando que há cansaço físico e mental, podendo gerar falta de atenção, estresse, desmotivação e ritmo acelerado de trabalho, com isso aumentando o risco da ocorrência de ATMB (MACHADO et al., 2015).

Quanto à remuneração, $21,7 \%$ dos enfermeiros têm faixa salarial entre 2.001 a 3.000 reais, contra $12,1 \%$ dos técnicos e auxiliares de enfermagem; $21,3 \%$ e $66,7 \%$ respectivamente tem faixa salarial abaixo de 2.000 reais; e $50 \%$ dos técnicos e auxiliares que recebem mais que 4.000 reais devido a vários vínculos empregatícios, conforme apresentado na figura 2. Em um estudo de Felli (2012) em hospitais universitários, 50,4\% dos profissionais de enfermagem tem renda de 1.001 a 2.000 reais, o que pode justificar a procura por outros vínculos empregatícios, fator que contribui para o aumento das horas semanais trabalhadas. Somam-se ainda cargas biológicas, psíquicas, mecânicas e químicas, a que estes profissionais estão expostos, aumentando a probabilidade do surgimento de doenças ocupacionais. Estes trazem consigo perdas econômicas $e$ humanas, $e$ às instituições empregadoras não compensam seus profissionais. É preciso repensar as condições de trabalho e a redução da jornada de trabalho para 30 horas a fim de melhorar a qualidade de vida no trabalho (FIOCRUZ, 2015). 


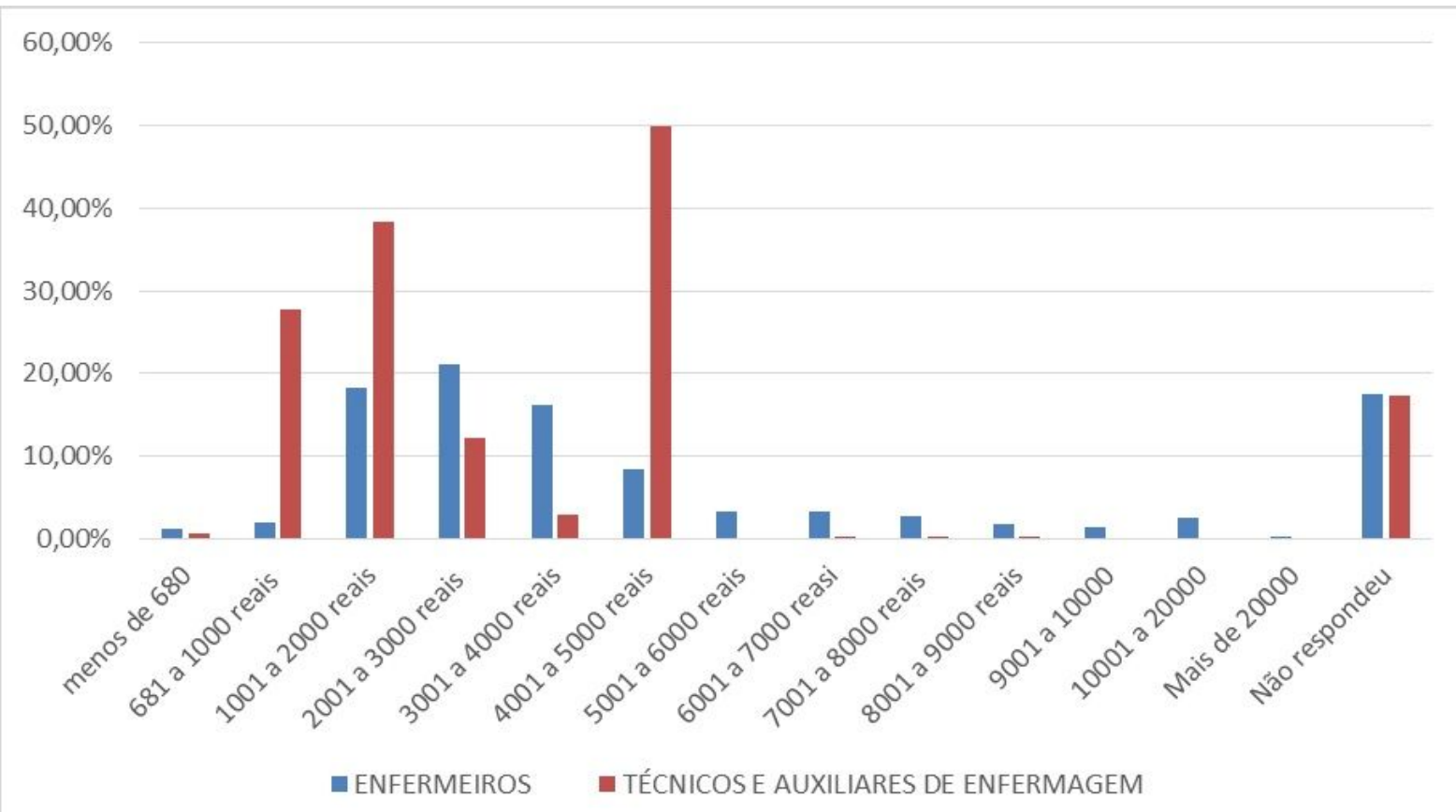

FIGURA 2: Renda mensal na atividade da equipe de enfermagem.

Fonte: Pesquisa Perfil da Enfermagem no Brasil - 2013. FIOCRUZ/COFEN

Os artigos analisados apontaram que o rendimento salarial mensal não é um fator predisponente para gerar o ATMB, mas consequências como, desmotivação, procura de mais de um emprego, ritmo de produção baixa e esgotamento de energia para a realização das tarefas. O rendimento salarial faz com que os profissionais da equipe da enfermagem se submetam a diferentes cargas de trabalho, podendo assim gerar adoecimentos, comprometimento a sua saúde e dos pacientes, e mais importante tornar pior a qualidade da assistência no hospital (FELLI, 2012).

Para Bakke e Araújo (2010), estes registros de ATMB despertam a precisão de políticas de segurança e saúde ocupacional desde a formação destes profissionais, pois as práticas que cumprirão no decorrer da vida laboral serão relembradas e aprendidas para melhor aprimoramento das medidas preventivas.

\section{Categoria profissional da equipe da enfermagem mais acometida pelo acidente de trabalho com exposição a material biológico}

No Brasil, a equipe da enfermagem é composta por três grupos de diferentes níveis de habilitação: o de nível superior, o enfermeiro; o de nível médio, o técnico de enfermagem e o auxiliar de enfermagem, nível de primeiro ou segundo grau. $\mathrm{O}$ enfermeiro coordena a equipe da enfermagem, e é responsável por planejar, programar, executar e avaliar as ações da enfermagem. O técnico de enfermagem auxilia o enfermeiro no seu planejamento, avaliação e execução de cuidados ao paciente. O auxiliar de enfermagem é o responsável por verificar, reconhecer e analisar sinais e sintomas de enfermidade do paciente, e prestar cuidados de higiene, auxílio e tratamentos simples; além de ajudar o enfermeiro e o técnico de enfermagem na prestação de assistência ao paciente (COFEN, 2007).

Algumas instituições ainda têm o atendente de enfermagem, que recebem uma capacitação pelas próprias instituições de saúde, tem a função de auxiliar os pacientes na alimentação, arrumar e manter a ordem do local de trabalho, lavar e preparar materiais para esterilização (COFEN, 2007). O enfermeiro é como um 
gerenciador e educador de risco, que tem o papel principal de orientar as ações preventivas com a finalidade de conscientizar a equipe sobre os possíveis riscos de AT em suas práticas profissionais (RODRIGUES et al., 2015). De acordo com a literatura analisada, os técnicos de enfermagem e auxiliares de enfermagem foram as categorias mais acometidas por ATMB, como pode ser constatado no Quadro 1.

QUADRO 1 - Distribuição de ATMB de acordo com a categoria da equipe da enfermagem.

\begin{tabular}{lcc}
$\begin{array}{c}\text { Autores de artigos } \\
\text { científicos }\end{array}$ & $\begin{array}{c}\text { Auxiliar de } \\
\text { enfermagem }\end{array}$ & $\begin{array}{c}\text { Técnico de } \\
\text { enfermagem }\end{array}$ \\
\hline Bakke e Araújo (2010) & $\mathrm{X}$ & $\mathrm{X}$ \\
\hline Rozario et al., 2009 & $\mathrm{X}$ & $\mathrm{X}$ \\
\hline Silva et al. (2010) & $\mathrm{X}$ & \\
\hline Araújo et al. (2012) & $\mathrm{X}$ & $\mathrm{X}$ \\
\hline Cavalcante et al. (2013) & & $\mathrm{X}$ \\
\hline Rezende et al. (2015) & & $\mathrm{X}$ \\
\hline Santos Jr. et al. (2015) & & $\mathrm{X}$
\end{tabular}

A maioria dos ATMB ocorreu com os técnicos de enfermagem, justifica-se pelo fato de ser este grupo de profissionais de maior contingente na área da saúde e que disponibilizam maior tempo para realizar suas atividades; pois prestam assistência direta aos pacientes, realizam diversos procedimentos invasivos e não invasivos (CAVALCANTE et al., 2013; ARANTES et al., 2017). Desta forma estes profissionais são mais vulneráveis à exposição a material biológico, e consequentemente a contrair doenças ocupacionais e ocorrência de acidente de trabalho (REZENDE et al., 2015; SANTOS et al., 2015).

Os técnicos de enfermagem manipulam muitos materiais como, agulhas e escalpes nas atividades de soroterapia e administração de medicamentos, o que pode levar ao acometimento de ATMB, sendo a maior categoria envolvida (CALVALCANTE et al., 2013). Já para Silva et al., (2010) e Araújo et al., (2012) os maiores envolvimentos em ATMB estão relacionados a categoria de auxiliar de enfermagem. Assim aponta-se que profissionais com menor conhecimento e qualificação podem estar perceptíveis a sofrerem mais ATMB do que os de nível técnico e superior. Estes profissionais estão envolvidos em atividades elementares, como: higiene de pacientes, conforto e transporte de pacientes, organização do ambiente e de documentos (ARAÚJO et al., 2012).

Para Becker e Oliveira (2008) as categorias profissionais da equipe da enfermagem com maior índice de absenteísmo são os auxiliares de enfermagem, correspondendo assim à função com a menor remuneração e exigência técnicocientífica e, comparando com as outras funções é a que tem a maior exigência física na atividade do cuidado ao paciente.

Outros autores como Rozario et al., (2009) , Bakke e Araújo (2010) e Oliveira, Gonçalves (2010) analisaram em seus estudos que as duas categorias tanto de técnico de enfermagem quanto auxiliar de enfermagem são as mais vistas nas estatísticas de ATMB. Isto se explica devido estas categorias desempenharem funções com maior envolvimento nas ocorrências de atendimento ao paciente, desenvolverem a maioria das atividades que são conferidas aos enfermeiros que os coordenam, e principalmente por conter o maior efetivo de profissionais entre a 
equipe da enfermagem. Então, com o objetivo de melhorar a formação destes profissionais e de suas práticas durante a vida laboral, deve-se implementar políticas de segurança e saúde ocupacional para diminuir estes acidentes de trabalho (NEGRINHO et al., 2017).

A categoria dos enfermeiros, os estudos mostraram que esta classe foi a menor envolvida nos ATMB; isto porque são considerados supervisores, chefes e se envolvem de maneira que tenha pouco contato com pacientes, consequentemente com materiais perfurocortantes e fluídos orgânicos (BAKKE; ARAÚJO, 2010; CAVALCANTE et al., 2013; ARANTES et al., 2017).

É importante destacar que as atividades da equipe da enfermagem, nas empresas hospitalares se caracterizam pela prestação de serviço ao paciente nas 24 horas do dia, implicando na permanência de grande parte da jornada de trabalho com o contato direto com o paciente (REZENDE et al., 2015).

Historicamente como diz Pedroza (2014), as categorias dos profissionais da equipe da enfermagem por não serem consideradas de alto risco para o acometimento de acidente de trabalho e doenças ocupacionais; os estudos voltaram para outro rumo confirmando que as unidades hospitalares são os locais de maior número de riscos ambientais (ocupacionais), consequentemente de ATMB.

Assim medidas para eliminar e/ou amenizar os ATMB é o investimento em prevenção, por meio de uma fiscalização preventiva e campanhas de conscientização para trabalhadores, as quais auxiliarão na inserção de uma cultura de prevenção às futuras gerações e que será capaz de reverter esse quadro preocupante no Brasil (SALIBA, 2018).

\section{CONSIDERAÇÕES FINAIS}

O acidente de trabalho com exposição a material biológico é considerado um problema de Saúde Pública, sendo preocupante para os empregadores e para o sistema previdenciário do Brasil devido aos seus efeitos nas vítimas deste tipo de acidente. Além disso, pode gerar prejuízos econômicos e sociais, isto porque o trabalhador acidentado, precisa afastar-se das suas atividades produtivas (REIS et al, 2013; GIANCOTTI et al., 2014; BRASIL, 2014; ARANTES et al., 2017).

Destaca-se que o ATMB ocorre na maioria das vezes em hospitais, que possui em atividade grande número de profissionais da equipe da enfermagem. Tais profissionais são na maioria do sexo feminino, na faixa etária entre 20 a 50 anos, estes realizam diversos procedimentos complexos, invasivos ou não, e possuem extensas jornadas de trabalho, assim tornam-se mais vulneráveis à ocorrência de agravos como, doenças ocupacionais e ATMB. Em relação a extensa jornada de trabalho observa-se que os profissionais da equipe da enfermagem que laboram acima de 40 horas semanais podem estar mais propícios a ocorrência de ATMB, pois demonstram cansaço físico e mental; podendo gerar desatenção, estresse, desmotivação e ritmo acelerado de trabalho.

O fator rendimento salarial mensal não foi observado como gerador de ATMB, mas nota-se entre os autores que consequências como: desmotivação, procura de mais de um emprego, ritmo de produção baixa e esgotamento de energia, podem gerar adoecimentos e comprometimento a saúde dos profissionais de saúde.

Os técnicos de enfermagem e auxiliares de enfermagem são as categorias mais acometidas por ATMB, isto porque as instituições hospitalares possuem um maior contingente destes profissionais para atender toda a assistência ao paciente e por serem estes profissionais que executam a maioria das atividades neste ambiente. Já a classe de enfermeiros não teve destaque nos envolvimentos aos 
ATMB, os mesmos são mais envolvidos em atividades na administração do setor da instituição hospitalar e tem poucos contatos com pacientes.

Podemos dizer ainda que os profissionais da equipe da enfermagem como os técnicos e auxiliares de enfermagem são mais acometidos pelo ATMB devido aos processos potencializadores de desgastes, como por exemplo, os cuidados permanentes, habituais e diretos com os pacientes. Desta forma, além de sérios comprometimentos a saúde também pode haver alteração na qualidade de vida.

Este trabalho de revisão de literatura nos possibilitou verificar através dos autores citados que o ambiente laboral pode propiciar diversos riscos a saúde dos profissionais da equipe da enfermagem e que diante de tantas dificuldades, interferências e trabalhos sob pressão, os ATMB são fáceis de ocorrer se não forem prevenidos imediatamente com ações de orientações e medidas de proteção variadas.

O caminho para diminuir os acidentes são as ações que devem ser implantadas como medidas de prevenção e orientações de segurança seguidas principalmente pela Norma regulamentadora de número 32 do Ministério do Trabalho, devem ser aplicadas para que estes acidentes sejam amenizados e/ ou eliminados desta categoria da área da saúde.

As instituições de saúde devem ser estruturadas sob uma visão de perspectiva do profissional e não somente no foco da produção e no ambiente de trabalho, ou seja, nas atividades rotineiras do local; realizando efetivamente limitações de cargas de trabalho e intervenções nos casos de surgimento de riscos que possam causar os ATMB e doenças ocupacionais.

Enfim é importante realizar educação continuada na área da saúde, intensificando adoção de práticas seguras que evitem os riscos ocupacionais e consequentemente ATMB. Esta pode ser de várias formas:palestras sobre biossegurança, cursos de prevenção (que podem ser tanto de aperfeiçoamento nas práticas laborais como também para as tecnologias avançadas de máquinas e equipamentos), rodas de conversas e principalmente capacitações de uso de equipamento de proteção individual. Tais metodologias aplicadas podem facilitar a sensibilização dos profissionais da equipe da enfermagem e a mudança de cultura das unidades hospitalares.

\section{REFERÊNCIAS}

ALVES, E. F. Características demográficas e ocupacionais do estudante-trabalhador de enfermagem e o risco de acidentes de trabalho. Trabalho \& Educação, v. 20, n. 3, p. 47-59, set-dez, $2011 . \quad$ Disponível em: <https://seer.ufmg.br/index.php/trabedu/article/view/7100/5475>. Acesso em 12 de jul. de 2018.

ARANTES, M. C.; HADDAD, M. C. F. L.; MARCON, S. S.; ROSSANEIS, M. A.; PISSINATI P. S. C.; et al. A. Acidentes de trabalho com material biológico em trabalhadores de serviços de saúde. Cogitare Enfermagem. Maringá, v. 22, n. 1, p.01-08, jan-mar, 2017. Disponível em: <http://dx.doi.org/10.5380/ce.v22i1.46508>. DOI: $10.5380 /$ ce.v22i1.46508

ARAÚJO, T. M.; CAETANO, A. J.; BARROS, L. M.; LIMA, A. C. F.; COSTA, R. M.; et al. Acidentes de trabalho com exposição a material biológico entre os profissionais de Enfermagem. Revista de Enfermagem Referência, III Série, n. 7, p. 7-14, Jul. 2012. Disponível em:<http://dx.doi.org/10.12707/RIII1182>. DOI: 10.12707/RIII1182. 
BAKKE, H. A., ARAÚJO, N. M. C. Acidentes de trabalho com profissionais de saúde de um Hospital Universitário. Produção. Paraíba, v. 20, n. 4, p. 669-676, out-dez., 2010. Disponível em: <http://dx.doi.org/10.1590/S0103-65132010005000015>. DOI: $10.1590 /$ S0103-65132010005000015

BARBOZA, D. B.; SOLER, Z. A. S. G.; CIORLIA, L. A. S. Acidentes de trabalho com pérfuro-cortante envolvendo a equipe de enfermagem de um hospital de ensino. Arquivo Ciências da Saúde, v. 11, n. 2, p. 2-8, abr-jun. 2004. Disponível em: $<$ http://repositorio-racs.famerp.br/racs_ol/Vol-11-2/ac07\%20-\%20id\%2047.pdf>.

Acesso em: 03 ago. de 2018.

BARBOSA, M. A., FIGUEIREDO, V. L., PAES, M. S. L. Acidentes de trabalho envolvendo profissionais de enfermagem no ambiente hospitalar: um levantamento em banco de dados. Revista Enfermagem Integrada, Ipatinga, v. 2, n. 1, p. 176187, jul-ago, 2009. ISSN 1981-1179. Disponível em: $<$ https://idonline.emnuvens.com.br/id/article/view/697/981>. Acesso em: 27 ago. de 2018.

BARROS, D. X.; TIPPLE, A. F. V.; LIMA, L. K. O. L.; SOUZA, A. C. S.; NEVES, Z. C. P.; SALGADO, T. A. Análise de 10 anos de acidentes com material biológico entre a equipe de enfermagem. Revista Eletrônica de Enfermagem. V. 18, 2016. ISSN 1518-1944. Disponível em: <http://dx.doi.org/10.5216/ree.v18.35493>. DOI: 10.5216/ree.v18.35493.

BECKER, S. G.; OLIVEIRA, M. L. C. Estudo do absenteísmo dos profissionais de enfermagem de um centro psiquiátrico em Manaus, Brasil. Revista LatinoAmericana Enfermagem, v. 16, n. 1, p. 109-114, jan-fev, 2008. ISSN 1518-8345. Disponível em: <http://dx.doi.org/10.1590/S0104-11692008000100017> DOI: 10.1590/S0104-11692008000100017

BEZERRA DE LIMA, M.; SALES DA SILVA, L. M.; MONTEIRO ALMEIDA, F. C.; TORRES, R. A. M.; DOURADO, H. M. H. Agentes estressores em trabalhadores de enfermagem com dupla ou mais jornada de trabalho. Revista de Pesquisa Cuidado é Fundamental Online, v. 5, n. 1, p. 3259-3266, Jan-mar, 2013. Disponível em: http://dx.doi.org/10.9789/2175-361.2013v5n1p3259. DOI: 10.9789/2175$5361.2013 v 5 n 1 p 3259$.

BRASIL. Ministério do Trabalho. Portaria n. 485, de 16 de novembro de 2005, NR32. Diário Oficial da República Federativa do Brasil, Brasília. Disponível em: <http://www.trtsp.jus.br/geral/tribunal2/ORGAOS/MTE/Portaria/P485_05.html>. Acesso: 04 de ago. de 2018.

Portal Brasil. Brasil e Alemanha discutem impacto dos acidentes de trabalho. [Internet] Brasília: MTE; 2014. Disponível em: <http://www.brasil.gov.br/economia-e-emprego/2014/03/brasil-e-alemanhadiscutem impacto-dos-acidentes-de-trabalho>. Acesso em: 03 de ago. de 2018.

Ministério da Previdência Social. Anuário estatístico da Previdência Social 2015. Brasília, 2015. Disponível em: <http://www.previdencia.gov.br/wpcontent/uploads/2015/08/AEPS-2015-FINAL.pdf>. Acesso em: 11 de ago. de 2018. 
Ministério da Previdência Social. Anuário estatístico de Acidente de Trabalho 2016. Brasília, 2016. Disponível em: <http://www.previdencia.gov.br/wpcontent/uploads/2016/08/AEPS-2016-FINAL.pdf>. Acesso em: 19 de ago. de 2018.

CAVALCANTE, C. A. A.; CAVAlCANTE, E. F. O.; MACÊDO, M. L. A. F.; CAVALCANTE, E. S.; MEDEIROS, S. M. Acidentes com material biológico em trabalhadores. Revista da Rede de Enfermagem do Nordeste. V. 14, n.4, p. 971979, mar. de 2013. ISSN 0303-7657. Disponível em: <http://dx.doi.org/10.1590/S0303-76572011000200011>. DOI: 10.1590/S030376572011000200011.

COFEN.- Conselho Federal de Enfermagem. Resolução n. $\mathbf{3 1 1}$ de 8 de fevereiro de 2007. Apresenta o Código de ética dos profissionais de enfermagem. Disponível em: <http://www.coren-rj.org.br/site/resolucoes/res_cofen_311-2007.pdf>. Acesso em: 12 ago. de 2018.

DONATELLI, S.; VILELA, R. A. G; ALMEIDA, I. M.; LOPES, M. G. R. Acidente com material biológico: uma abordagem a partir da análise das atividades de trabalho. Saúde Sociedade. São Paulo, v.24, n.4, p.1257-1272, 2015. Disponível em: <http://dx.doi.org/10.1590/S0104-12902015136790>. DOI: 10.1590/S010412902015136790.

FELLI, V. E. A. Condições de trabalho de enfermagem e adoecimento: motivos para a redução da jornada de trabalho para 30 horas. Enfermagem em Foco, v. 3, n. 4, p. 178-181, 2012. Disponível em: <https://doi.org/10.21675/2357707X.2012.v3.n4.379> DOI: 10.21675/2357-707X.2012.v3.n4.379

FIOCRUZ. Pesquisa inédita traça perfil da enfermagem no Brasil. Fundação Oswaldo Cruz. Rio de Janeiro: 2015. Disponível em: <http://portal.fiocruz.br/ptbr/ content/pesquisa-inedita-traca-perfil-da-enfermagem-no-brasil>. Acesso em: 27 ago. de 2018.

GIACONTTI, G. M.; HAEFFNER, R.; SOLHEID, N. L. S.; MIRANDA, F. M. A.; SARQUIS, L. M. M. Caracterização das vítimas e dos acidentes de trabalho com material biológico atendidas em um Hospital público do Paraná, 2012. Epidemiologia e Serviço Saúde. Brasília, v. 23, n. 2, p. 337-346, abr-jun, 2014. Disponível em: <https://doi.org/10.5123/S1679-49742014000200015>. DOI: $10.5123 /$ S1679-49742014000200015

KON, N. M.; SOLTOSKI, F.; REQUE, JR. M.; LOZOVEY, J. C. A. Acidentes de trabalho com material biológico em uma Unidade Sentinela: casuística de 2.683 casos. Revista Brasileira Medicina do Trabalho, v. 9, n. 1, p. 33-38, 2011. Disponível em: <file:///C:/Users/Usuario/Downloads/v9n1a05\%20(4).pdf>. Acesso em 12 de jul. de 2018.

MACHADO, J. M. H.; ASSUNÇÃO, A. A. Panorama da saúde dos trabalhadores da saúde. Belo Horizonte: UFMG, 2012. Disponível em: <https://site.medicina.ufmg.br/nest/wpcontent/uploads/sites/33/2015/04/Panorama_e -book.pdf>. Acesso em: 10 de mai. de 2017. 
MACHADO, M. H.; AGUIAR, W. F.; LACERDA, W. F.; OLIVEIRA, E. W.; LEMOS,W. M.; et al. Relatório final da Pesquisa Perfil da Enfermagem no Brasil (Convênio: Fiocruz/Cofen). Rio de Janeiro: 28 volumes, NERHUS-DAPS-Ensp/Fiocruz e Cofen; 2015. Disponível

em: $<$ http://revista.cofen.gov.br/index.php/enfermagem/article/view/686/296>. Acesso em: 27 ago. de 2018.

MARTINS, R. J.; MOIMAZ, S. A. S.; GARBIN, A. J. Í.; GONÇALVES, P. R. V.; GARBIN, C. A. S. Prevalência de Acidentes com material biológico em um município do noroeste de São Paulo, Brasil, no período de 2007 a 2011. Ciencia \& Trabajo, ano 16, n.50, p. 93-96, Mai-Ago, 2014. Disponível em: <http:https://scielo.conicyt.cl/pdf/cyt/v16n50/art06.pdf>. Acesso em 12 de agosto de 2018.

MARZIALE, M. H. P.; SANTOS, H. E. C.; CENZI, C. M.; ROCHA, F. L. R.; TROVÓ, M. E. M. Consequências da exposição ocupacional a material biológico entre trabalhadores de um hospital universitário. Escola Anna Nery Revista de Enfermagem. São Paulo, v. 18, n. 1, p. 11-16, Jan-Mar, 2014. Disponível em: <https://doi.org/10.5935/1414-8145.20140002>. DOI: 10.5935/1414-8145.20140002

NEGRINHO, N. B. S.; MALAGUTI-TOFFANO, S. E.; REIS, R. K.; PEREIRA, F. M. V.; GIR, E. Fatores associados a exposição ocupacional com material biológico entre profissionais de enfermagem. Revista Brasileira de Enfermagem. v. 70, n. 1, p. 133-138, Jan-Fev, 2017. Disponível em: <https://doi.org/10.1590/0034-7167-20160472>. DOI: 10.1590/0034-7167-2016-0472

NOGUEIRA, S. A.; CARVALHO, B. K. G.; MEDEIROS, A. R.; CARNEIRO, S. E. R.; SOUZA, G. C. A. Prevalência e notificações de Acidentes de Trabalho com exposição a material biológico na Odontologia. Revista Ciência Plural. V. 2, n. 1, p. 102-119, $2016 . \quad$ Disponível em: <https://periodicos.ufrn.br/rcp/article/view/10040/7137>. Acesso em 09 de ago. de 2018.

NOWAK, N. L.; CAMPOS, G. A.; BORBA, E. O.; ULBRICHT, L.; NEVES, E. B. Fatores de risco para acidentes com materiais perfurocortantes. O Mundo da Saúde. São Paulo, v. 37, n. 4, p. 419-426, 2013. Disponível em: $<$ https://www.saocamilo-sp.br/pdf/mundo_saude/155558/A06.pdf>. Acesso em: 23 de ago. de 2018.

OLIVEIRA, A. C.; GONÇALVES, J. A. Acidente ocupacional por material perfurocortante entre profissionais de saúde de um Centro Cirúrgico, Revista da Escola de Enfermagem. São Paulo, v. 44, n. 2, p. 482-487, 2010. Disponível em: $<$ http://www.scielo.br/pdf/reeusp/v44n2/34.pdf>. Acesso em 10 de jul. de 2018.

Organização Internacional do Trabalho. ONUBR, 2018. Disponível em: <https://nacoesunidas.org/agencia/oit/>. Acesso em: 15 março de 2018.

PAIVA, S. N.; ZARONI, W. C. S.; LEITE, M. F.; BIANCHI, P. R.; PEREIRA, T. C. R. Acidentes ocupacionais com material biológico em Odontologia: uma responsabilidade no ensino. Revista da ABENO, v. 17, n. 3, p. 76-88, 2017. 
Disponível em: $\quad$ http://dx.doi.org/10.30979/rev.abeno.v17i3.388>. DOI: 10.30979/rev.abeno.v17i3.388.

PEDROZA, M. G. S. Riscos ocupacionais do trabalho de profissionais de Enfermagem. Revista Especialize On-line IPOG. Goiânia, 7ª Edição, v. 01, n. 07, Julho, 2014. ISSN 2179-5568. Disponível em: <https://www.ipog.edu.br/revistaespecialize-online/edicao-n7-2014/riscos-ocupacionais-do-trabalho-de-profissionaisde-enfermagem/>. Acesso em: 03 de set. de 2018.

RAFFONE, A. M.; HENNINGTON, E. A. Avaliação da capacidade funcional dos trabalhadores de enfermagem. Revista Saúde Pública. v. 39, n. 4, 2005. Disponível em: <http://dx.doi.org/10.1590/S0034-89102005000400023>. DOI: 10.1590/S003489102005000400023.

REIS, P. G. T. A.; DRIESSEN, A. N.; COSTA, A. C. B. A.; NASR, A.; COLLAÇO, I. A.; et al. Perfil epidemiológico de acidentes com material biológico entre estudantes de medicina em um pronto-socorro cirúrgico. Revista do Colégio Brasileiro de Cirurgiões. São Paulo, v. 40, n. 4, p. 287-292, 2013. Disponível em: <http://www.scielo.br/pdf/rcbc/v40n4/v40n4a06.pdf>. Acesso em 19 de set. de 2018.

REZENDE, L. C. M.; LEITE, K. N. S.; DOS SANTOS, S. R., MONTEIRO, L. C.; COSTA, M. B. DE S.; et al. Acidentes de trabalho e suas repercussões na saúde dos profissionais de enfermagem. Revista Baiana de Enfermagem, Salvador, v. 29, n. 4, p. 307-317, out./dez. 2015. Disponível em: <http://dx.doi.org/10.18471/rbe.v29i4.13559> DOI: 10.18471/rbe.v29i4.13559.

RIOS, M. A.; NERY, A. A.; ALVES, M. S.; JESUS, C. S. Acidentes e doenças relacionadas ao trabalho em Jequié, Bahia, registrados no Instituto Nacional de Seguridade Social. Epidemiologia Serviço Saúde. Brasília, v. 21, n. 2, p. 315-324, abr-jun., 2012. Disponível em: <http://dx.doi.org/10.5123/S167949742012000200014>. DOI: 10.5123/S1679-49742012000200014

RODRIGUES, F. M. S.; NOGUEIRA-JUNIOR, C.; AMARAL, E. M. S.; FERNANDES, Â. C. P. Notificação de acidentes de trabalho com perfurocortantes: experiências de uma equipe de enfermagem. Revista de Enfermagem. Juiz de Fora, v. 1, n. 2, p. 145-152, jul-dez, 2015. Disponível em: < https://enfermagem.ufjf.emnuvens.com.br/enfermagem/article/view/24>. Acesso em: 09 de ago. de 2018.

ROZARIO, S.; SILVA, J L L; TEIXEIRA, E. R.; COSTA, S. F.; FARIA, A. L. F.; MACEDO, C. A. Acidentes com Pérfuro-Cortantes na equipe de Enfermagem. Revista de enfermagem UFPE. v. 3, p. 291-298, 2009. Disponível em: $<$ www.ufpe.br/revistaenfermagem/index.php/revista/article/.../2948>. Acesso em: 09 de mai. de 2018.

SALIBA, T. M. Manual Prático de Higiene Ocupacional e PPRA. 4aㅡ. Ed - São Paulo: LTr, fev. 2013. 368p.

SALIBA, T. M. Manual Prático de Higiene Ocupacional e PPRA. 6a․ Ed - São Paulo: LTr, mai. 2015. 496p. 
SALIBA, T. M. Manual Prático de Higiene Ocupacional e PPRA. 8a․ Ed - São Paulo: LTr, jan. 2017. 252p

SALIBA, T. M. Curso Básico de Segurança e Higiene Ocupacional. 8ª . Ed - São Paulo: LTr, fev. 2018. 494p.

SANTOS Jr., E. P.; BATISTA, R. R. A. M.; ALMEIDA, A. T. F.; ABREU, R. A. A. Acidente de trabalho com material perfurocortante envolvendo profissionais $\mathrm{e}$ estudantes da área da saúde em hospital de referência. Revista Brasileira Medicina do Trabalho. v. 13, n. 2, p. 69-75, 2015. Disponível em: <http://www.anamt.org.br/site/upload_arquivos/rbmt_volume_13_n\%C2\%BA_2_2932 0161552145795186.pdf>. Acesso em: 02 de jul. de 2018.

SILVA, J. A.; PAULA, V. S.; ALMEIDA, A. J.; VILLAR, L. M. Investigação de acidentes biológicos entre profissionais de saúde. Escola Anna Nery. Revista de Enfermagem. V. 13, n. 3, p. 508-516, jul-set, 2009. Disponível em: <http://www.scielo.br/pdf/ean/v13n3/v13n3a08>. Acesso em: 05 de jun. de 2018.

SILVA, T. R.; ROCHA, S. A.; AYRES, J. A.; JULIANI, C. M. C. M. Acidente com material perfurocortante entre profissionais de enfermagem de um hospital universitário. Revista Gaúcha Enfermagem, Porto Alegre. v. 31, n. 4, p. 615-622, dez, 2010.2 Disponível em: <http://seer.ufrgs.br/RevistaGauchadeEnfermagem/article/view/14705/11841>.

Acesso em: 09 de jul. de 2018.

SINAN - Sistema de Informação de Agravos de Notificação.Estatística Dos Agravos Relacionados ao Trabalho. Acidente Com Exposição a Material Biológico. Secretaria de Estado da Saúde Pública. Centro Estadual de Referência em Saúde do Trabalhador [Internet]. 2011 [cited 2017 Nov 25]. Disponível em: $<$ http://www.cerest.rn.gov.br/contentproducao/aplicacao/sesap_cerest/cerest/agravos ces_rn/estatistic_sinan\%20rn\%20acbio-ok.pdf>. Acesso em: 09 de mai. de 2018. 